\title{
Low-noise transfer of the spectral purity of an optical comb line using a feedforward scheme
}

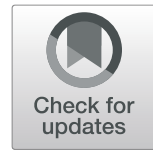

\author{
Pierre Brochard ${ }^{1 *} \mathbb{D}$, Benjamin Rudin², Florian Emaury², Valentin J. Wittwer ${ }^{1}$, Stéphane Schilt ${ }^{1}$ and \\ Thomas Südmeyer ${ }^{1}$
}

\begin{abstract}
We present a detailed frequency noise analysis of a feedforward scheme used to faithfully transfer the spectral properties of an individual line of an optical frequency comb spectrum to a single-mode laser and in this way indirectly amplify it, which is applicable to any arbitrary comb mode spacing. In contrast to previously reported implementation of the feedforward method for a similar purpose, we present a more thorough noise study, including the measurement of the additive noise of the setup. The reported experimental investigation was performed using two low-noise ultrafast mode-locked lasers with different repetition rates $(\sim 1 \mathrm{GHz}$ and $250 \mathrm{MHz}$ ) and show a faithful transfer of the comb mode frequency noise to the auxiliary laser with a sub-radian additive phase noise integrated from $1 \mathrm{~Hz}$ to $1 \mathrm{MHz}$. We discuss the present limitations of the method that is able to transfer optical comb lines with sub- $\mathrm{Hz}$ linewidth and propose simple improvements.
\end{abstract}

Keywords: Feedforward, Optical frequency comb, Frequency noise

\section{Introduction}

Optical frequency combs (OFCs) from femtosecond modelocked lasers represent a powerful tool in time and frequency metrology as they provide a direct and phasecoherent link between the optical and microwave spectral domains. Self-referenced combs deliver absolute optical frequencies that benefit many research fields [1, 2]. Among the variety of applications of frequency combs, some make essentially use of only one particular component of the comb spectrum. This is the case for instance for optical frequency references for differential absorption lidar (DIAL) systems or for optical frequency synthesizers. Moreover, they also require a high optical power in this specific mode, which is most often not directly available from an OFC. Furthermore, extracting and amplifying a single line of a comb with a sub-GHz mode spacing is rather challenging, as no suitable conventional optical filter (e.g., based on thin films or gratings) with a sufficiently narrow bandwidth is commercially available. A possible

\footnotetext{
* Correspondence: pierre.brochard@unine.ch

'Laboratoire Temps-Fréquence, Institut de Physique, Université de Neuchâtel, CH-2000 Neuchâtel, Switzerland

Full list of author information is available at the end of the article
}

solution to this issue may be to first enlarge the comb mode-spacing to the multi-GHz range using a pulse repetition rate multiplier (PRRM) as frequently used in ultralow-noise microwave generation by optical-to-microwave frequency division realized with fs-laser frequency comb $[3,4]$. This can be achieved either with a combination of Fabry-Perot optical cavities of different free spectral ranges [3] or with an interleaver system made of a series of fibered or free-space unbalanced Mach-Zehnder interferometers [4]. After repetition rate multiplication, a line of the resulting comb can be isolated more easily using a conventional optical bandpass filter. However, this makes the system much more complex and poorly tunable if the comb line to be extracted needs to be switchable.

Therefore, other methods have been developed to isolate and amplify a single frequency component from a comb spectrum. The most direct approach is to phaselock an auxiliary single-mode laser to the comb mode. The method is similar to a concept commonly used in comb-assisted spectroscopy, where a single-mode tunable laser is locked to a mode of an OFC to accurately scan an absorption line of a molecular species [5]. However, a fast frequency control of the auxiliary laser is 
needed to properly imprint the comb line spectral properties to the scanning laser. Injection locking of a continuous-wave (cw) laser by the comb mode of interest is an alternative method to extract a single comb mode that offers such a high control bandwidth. It has been successfully reported for OFCs with mode spacing of $10-\mathrm{GHz}$ or higher [6] as well as with sub-GHz repetition rate combs $[7,8]$ and down to $56 \mathrm{MHz}$ using a frequency-selective Brillouin amplifier that amplifies an individual comb line while efficiently suppressing the others [9]. Under injection locking, the auxiliary laser acquires the spectral properties of the comb mode and constitutes the desired filtered and amplified output signal. Injection locking is fairly direct to implement with GHz-range mode spacing combs, but becomes more challenging with low repetition rate OFCs as different comb modes may lay within the injection locking range of the auxiliary laser.

The feedforward method is an alternative approach to transfer the spectral purity of a comb line to an auxiliary single-mode laser [10]. It uses feedforward corrections applied to a frequency shifter modulator (generally an acousto-optic frequency shifter - AOFS) to compensate for the frequency fluctuations between the laser and the comb line. The method was first introduced to phaselock the carrier-envelope offset (CEO) frequency $f_{\text {CEO }}$ of a Ti:sapphire optical frequency comb with a high bandwidth [11]. Feedforward stabilization was also recently applied to stabilize the CEO frequency in other types of frequency combs with the demonstration of ultralow noise performance, such as in a Yb:CYA laser [12] or in an Er:Yb:glass laser [13]. In this article, we show a detailed noise analysis of the feedforward method used to transfer the spectral purity of a selected comb line to an auxiliary laser, including the measurement of the additive noise of the implemented setup that determines its ultimate capability. Such an analysis was not presented before in previous implementations of a feedforward scheme for this purpose, i.e., neither in the work of Sala et al. [10], nor in the analysis of the method presented by Gatti et al. [14]. Our investigations made with two OFCs of different repetition rates $(\sim 1 \mathrm{GHz}$ and 250
$\mathrm{MHz}$ ) show that the additive phase noise of the setup is around $0.5 \mathrm{rad}$, integrated from $1 \mathrm{~Hz}$ to $1 \mathrm{MHz}$, which demonstrates that a sub- $\mathrm{Hz}$ relative linewidth between the selected comb line and the output signal can be achieved, meaning that a Hz-level linewidth comb mode can be faithfully transferred to the output laser. This statement complements very recent results published in parallel to our study on the feedforward stabilization of a low-noise 1064-nm Nd:YAG laser to a Yb:fiber frequency comb referenced to an ultra-stable laser [15]. The study that we report here was made in a different noise regime and we present a more detailed noise analysis of the feedforward scheme with a different approach to assess the additive noise of the setup.

\section{Methods/experimental}

Our experimental study was realized using two commercial low-noise mode-locked femtosecond lasers (model MENHIR-1550 from Menhir Photonics AG), without any active stabilization in this work. Both lasers emit clean soliton $\operatorname{sech}^{2}$-pulses in a diffraction-limited beam delivered via a fibered output port. The first modelocked laser (which is referred to as OFC-1) has a repetition rate of $\sim 1 \mathrm{GHz}$ and the second (OFC-2) has a lower repetition rate of $250 \mathrm{MHz}$. The detailed parameters of these two combs are summarized in Table 1.

Both mode-locked lasers were operated in freerunning mode in this experiment. This was motivated by the fact that no detection of CEO frequency by octavespanning supercontinuum spectrum generation and subsequent nonlinear interferometry [16] was implemented with these lasers at the time of the experiment to selfreference them. Another reason is that these lasers show already a very low phase noise in free-running mode in comparison to typical $250-\mathrm{MHz}$ fiber combs, especially at high offset frequencies as illustrated by their phase noise power spectral density displayed in Fig. 1. The phase noise was measured for both lasers at the $10-\mathrm{GHz}$ harmonic of the repetition rate using a photodiode with a highly-linear response (model DSC40S from Discovery Semiconductors Inc.) and a cross-correlator phase noise analyzer (Rohde-Schwarz FSWP-26). The corresponding

Table 1 Main parameters of the used commercial mode-locked lasers

\begin{tabular}{lll}
\hline Parameters & OFC-1 & OFC-2 \\
\hline Repetition rate & $\sim 1 \mathrm{GHz}(975.5 \mathrm{MHz})$ & $250 \mathrm{MHz}$ \\
Average output power & $35 \mathrm{~mW}$ & $90 \mathrm{~mW}$ \\
Center wavelength & $1560 \mathrm{~nm}$ & $1556 \mathrm{~nm}$ \\
Optical bandwidth (at $-3 \mathrm{~dB})$ & $>13 \mathrm{~nm}$ & $>13 \mathrm{~nm}$ \\
Supported pulse duration & $<200 \mathrm{fs}$ & $<200 \mathrm{fs}$ \\
Free-running timing jitter $[1 \mathrm{kHz}-10 \mathrm{MHz}]$ & $<10 \mathrm{fs}$ & $<1.5 \mathrm{fs}$ \\
Free-running 3-dB linewidth of an optical comb line $[1 \mathrm{~Hz}-10 \mathrm{MHz}]$ & $<18 \mathrm{kHz}$ & $<10 \mathrm{kHz}$ \\
\hline
\end{tabular}



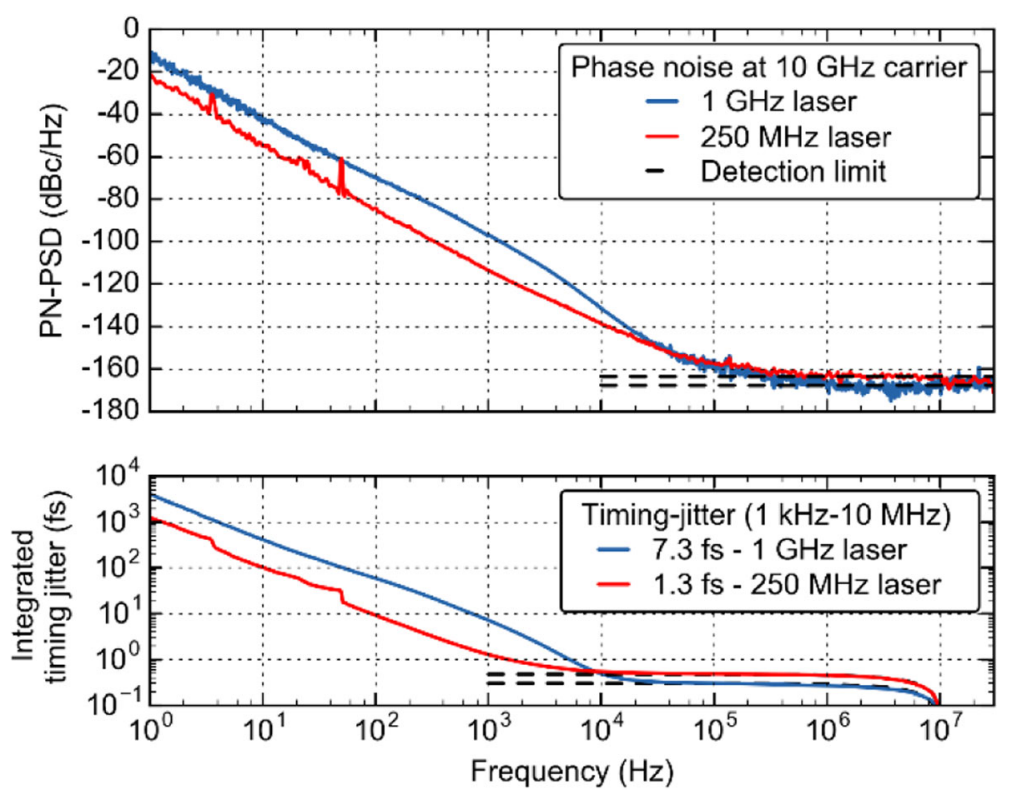

Fig. 1 (Top) Phase noise power spectral density (PN-PSD) of the two commercial mode-locked lasers used in the experiment measured at a high harmonic of the repetition rate at around $10 \mathrm{GHz}$. (Bottom) Corresponding integrated timing jitter (integration from $1 \mathrm{kHz}$ to $10 \mathrm{MHz}$ ). The dashed black lines indicate the noise floor of the measurement

timing jitter is below $10 \mathrm{fs}$ for the $1-\mathrm{GHz}$ repetition rate comb and in the range of $1 \mathrm{fs}$ for the $250-\mathrm{MHz}$ comb (integration from $1 \mathrm{kHz}$ to $10 \mathrm{MHz}$ ). The linewidth (full width at half maximum - FWHM) of an optical mode measured at $\sim 1557.3 \mathrm{~nm}$ is around $17.5 \mathrm{kHz}$ for the $\mathrm{GHz}$ comb and only $9.3 \mathrm{kHz}$ for the $250-\mathrm{MHz}$ comb at 1 -s integration time. These values were obtained from the frequency noise power spectral density (FN-PSD) measured for the heterodyne beat with an ultra-narrowlinewidth reference laser using the $\beta$-separation line approximation [17].

The implemented feedforward scheme illustrated in Fig. 2 consists of imprinting the phase noise of the selected comb line to an auxiliary CW laser by removing at the same time the own phase noise of this $\mathrm{CW}$ laser. This is realized by detecting the heterodyne beat-note between the comb mode and the auxiliary laser and by applying it to drive an AOFS (model FCM- 401E6AP from IntraAction Corp.), as described in the green area in Fig. 2. The CW auxiliary laser (model ORION from Redfern Integrated Optics) emits at $\sim 1557 \mathrm{~nm}$ with an optical power of around $8 \mathrm{~mW}$ and a linewidth in the range of a few tens of kilohertz. The laser output power is first split into two parts using a 50:50 fibered splitter. The first part is combined with the optically-filtered (1$\mathrm{nm}$ bandwidth) commercial low-noise mode-locked laser (OFC-1 or OFC-2) and sent to a standard InGaAs photodiode in order to generate an optical beat note at around $40 \mathrm{MHz}$ (operation frequency of the AOFS). The beat note is then amplified to drive the AOFS that modulates the second part of the laser output. As the beat-note signal contains the difference between the frequency fluctuations of the comb mode and of the auxiliary laser, the frequency fluctuations of the auxiliary laser cancel out in the up-shifted frequency at the output of the AOFS (provided that the correct sign of the beat signal is used), while the frequency noise of the comb mode is imprinted onto this signal. Therefore, the signal at the output of the AOFS constitutes an amplified copy (with a power of several $\mathrm{mW}$ ) of the selected comb mode. In this process, extra noise in the beat signal between the comb line and the auxiliary laser (e.g., shot-noise, amplitude to phase noise conversion, radio-frequency amplifier noise) must be minimized not to deteriorate the resulting phase noise of the filtered comb line.

\section{Results and discussion}

The measured frequency noise of the initial comb mode and of its amplified and filtered version (feedforward output) is displayed in Fig. 3. The frequency noise of the initial optical comb line was assessed from its heterodyne beat note with a CW diode laser (model PLANEX from Redfern Integrated Optics) stabilized to an ultralow expansion (ULE) optical cavity and was measured using a phase noise analyzer (FSWP-26 from RohdeSchwarz) as shown in the red area in Fig. 2. Despite the $\mathrm{Hz}$-level linewidth of this reference laser, it was the limiting factor in the frequency noise of the optical beat note at high Fourier frequencies (resulting notably from the servo-bump at $\sim 100 \mathrm{kHz}$ of its stabilization to the 


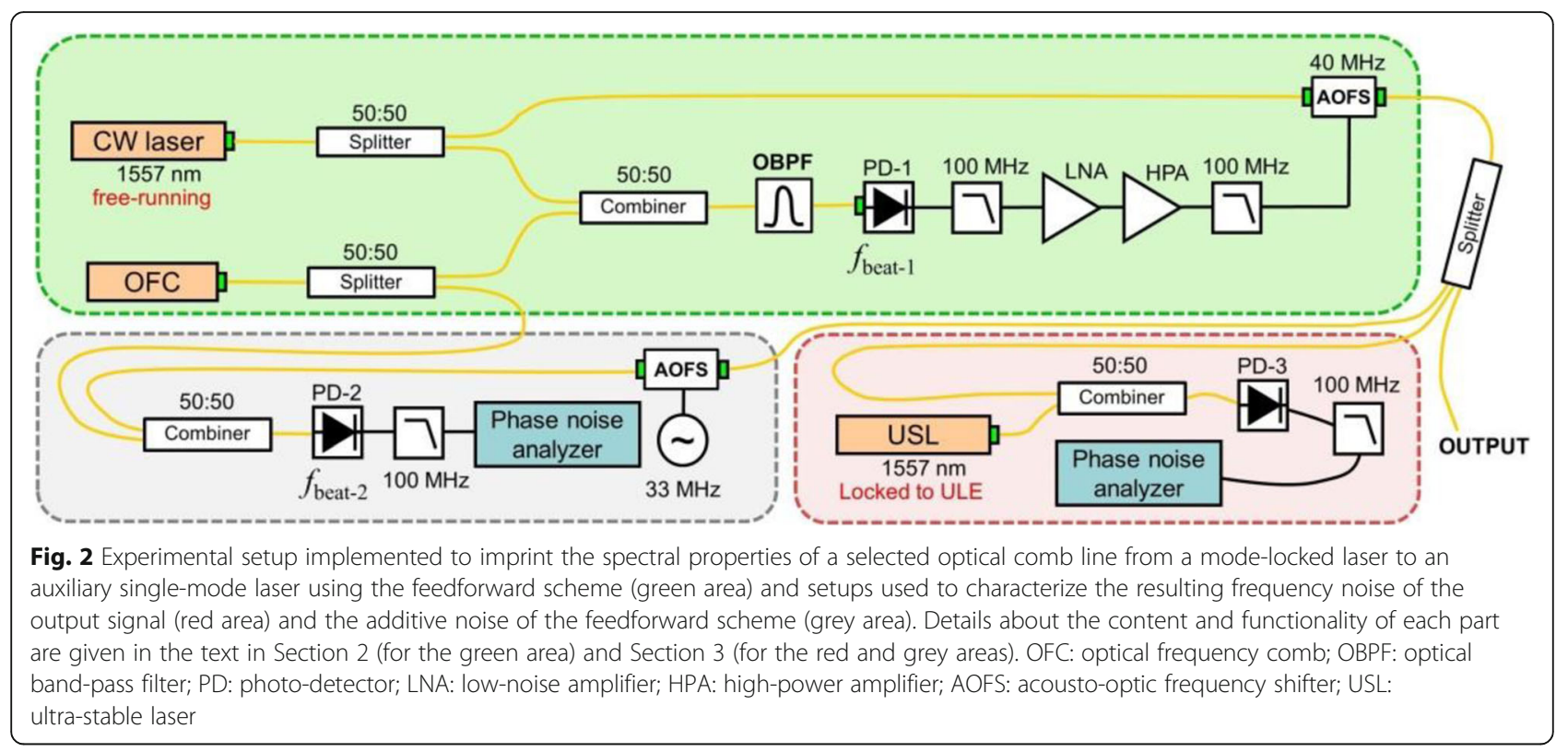

optical cavity as well as from its white frequency noise plateau). This demonstrates the very low-noise properties of the used mode-locked lasers even without any active stabilization.

The additive frequency noise of the feedforward scheme was also separately characterized. It cannot be assessed from the simple subtraction of the FN-PSD measured for the initial comb line and transferred to the auxiliary laser, as these two curves are very close to each other at a fairly high noise level, so that their difference would be very sensitive to any small measurement imprecisions. Therefore, a dedicated measurement was implemented to directly assess the additive noise. For this purpose, the output signal was frequency-shifted by another AOFS (driven by a synthesizer with a negligible noise) and was optically combined with the comb mode in order to remove its noise contribution (grey area in Fig. 2). The additive noise of the setup was assessed from the frequency noise of this heterodyne beat signal. It clearly shows that the additive noise is completely negligible at low Fourier frequencies. It becomes dominant at Fourier frequencies higher than $\sim 10 \mathrm{kHz}$ here (Fig. 3, grey curve), which depends on the initial noise of the auxiliary laser. The integrated phase noise of the additive noise calculated in the range of $1 \mathrm{~Hz}-1 \mathrm{MHz}$ is $550 \mathrm{mrad}$, which demonstrates that the studied feedforward scheme can transfer Hz-level linewidth optical comb lines. The limited bandwidth of the used AOFS induces a noise bump at around $500 \mathrm{kHz}$, which is the dominant limitation identified in this study as also observed in the work of Gatti et al. [12]. Further noise minima and maxima occur at even and odd harmonics

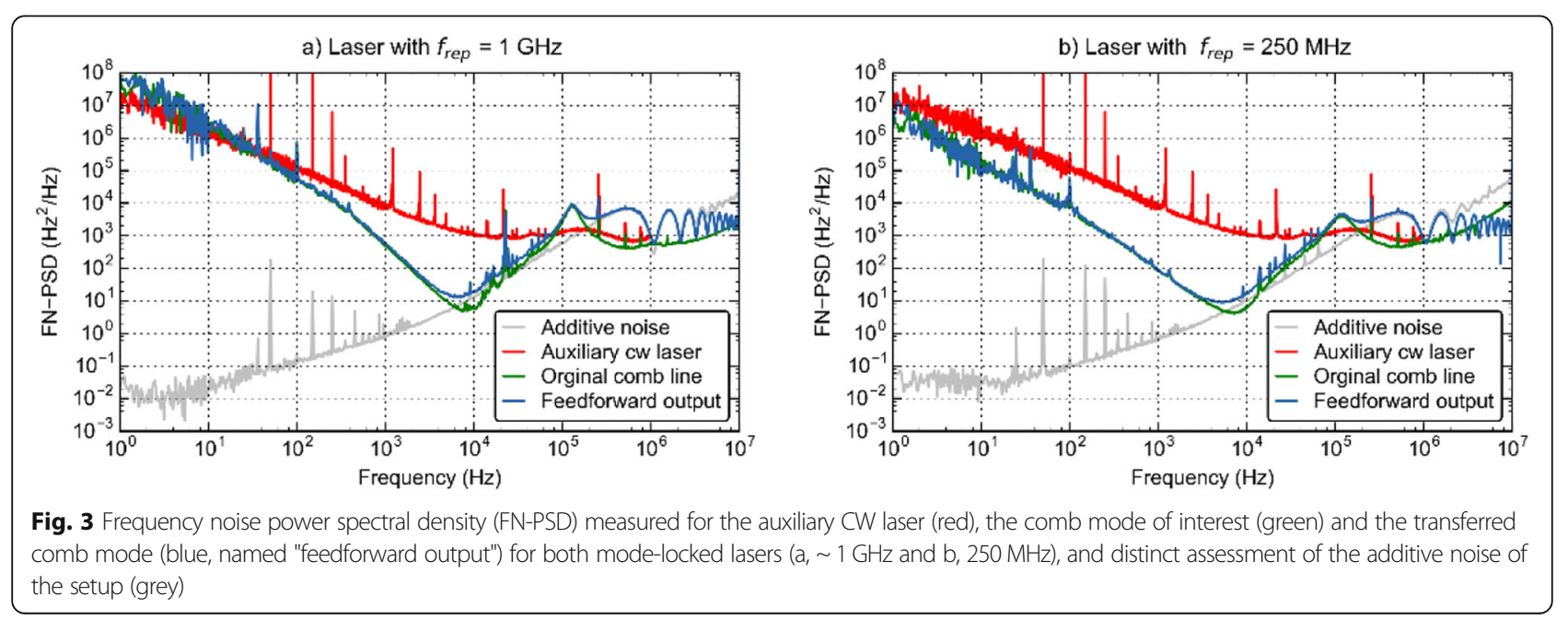


of the AOFS bandwidth. The maxima take place when the phase shift induced by the AOFS is an odd multiple of $180^{\circ}$, whereas minima occur at even harmonics. A modulation bandwidth of $500 \mathrm{kHz}$ was separately assessed for this AOFS from a measurement of its modulation transfer function. Faster AOFSs with a modulation bandwidth $>10 \mathrm{MHz}$ that are commercially available or the use of a single-sideband electro-optics modulator (EOM) [18] can improve the performance of the proposed feedforward method by enlarging the bandwidth in which the noise properties of the comb mode are faithfully transferred to the auxiliary laser.

\section{Conclusion}

We have presented a detailed frequency noise study of a feedforward scheme used to faithfully transfer the spectral properties of a single optical line of an OFC to an auxiliary laser and characterized the performances of the method with its current limitations. The additive noise analysis of the setup demonstrates its ability to simultaneously copy and amplify an optical comb line at the sub$\mathrm{Hz}$ linewidth operation. The main limitation arises from the bandwidth of the used AOFS and the initial frequency noise spectrum of the auxiliary laser. The use of a faster AOFS or a single-side band electro-optic modulator (SSB-EOM) [18] as well as of an auxiliary laser with a lower frequency noise (especially at high Fourier frequencies), like a fiber laser, will further improve the performance of the method by transferring the noise properties of the OFC mode in a wider bandwidth.

\section{Abbreviations \\ AOFS: Acousto-Optic Frequency Shifter; CEO: Carrier-Envelope Offset; CW: Continuous-Wave; DIAL: Differential Absorption Lidar; EOM: Electro-Optic Modulator; FN-PSD: Frequency Noise Power Spectral Density; FWHM: Full Width at Half Maximum; HPA: High Power Amplifier; LNA: Low Noise Amplifier; OBPF: Optical Band-Pass Filter; OFC: Optical Frequency Comb; PD: Photo-Detector; PN-PSD: Phase Noise Power Spectral Density; PRRM: Pulse Repetition Rate Multiplier; SSB-EOM: Single Side-Band Electro- Optic Modulator; ULE: Ultra-Low Expansion; USL: Ultra-Stable Laser}

\section{Acknowledgements}

Not applicable.

\section{Authors' contributions}

PB and FE performed the experimental work with assistance of VJW and SS BR designed and built two mode-locked lasers used in this work. PB and FE analyzed the data and wrote the manuscript with SS and inputs from the other authors. TS supervised the project. All authors read and approved the final manuscript.

\section{Funding}

BRIDGE CombTrace (grant 20B2-1_176584), ESA-BIC Switzerland.

\section{Availability of data and materials}

The data of the results reported in this manuscript will be available on Zenodo upon publication of the article. Experimental results presented in this work are open-access available under DOl: http://doi.org/10.23728/b2 share.64fb65affbe144d4823ff84936b0df41.

\section{Competing interests}

The authors declare that they have no competing interests.

\section{Author details}

'Laboratoire Temps-Fréquence, Institut de Physique, Université de Neuchâtel, CH-2000 Neuchâtel, Switzerland. ${ }^{2}$ Menhir Photonics AG, Thiersteinerallee 71, CH-4053 Basel, Switzerland.

Received: 11 November 2019 Accepted: 27 January 2020

Published online: 07 February 2020

References

1. Hänsch, T.W.: Nobel lecture: passion for precision. Rev. Mod. Phys. 78(4), 1297-1309 (2006)

2. Hall, J.L.: Nobel lecture: defining and measuring optical frequencies. Rev. Mod. Phys. 78(4), 1279-1295 (2006)

3. Diddams, S.A., Kirchner, M., Fortier, T., Braje, D., Weiner, A.M., Hollberg, L.: Improved signal-to-noise ratio of $10 \mathrm{GHz}$ microwave signals generated with a mode-filtered femtosecond laser frequency comb. Opt. Express. 17(5), 3331-3340 (2009)

4. Haboucha, A., Zhang, W., Li, T., Lours, M., Luiten, A.N., Coq, Y.L., Santarelli, G.: Optical-fiber pulse rate multiplier for ultralow phase-noise signal generation. Opt. Lett. 36(18), 3654-3656 (2011)

5. Gambetta, A., Gatti, D., Castrillo, A., Coluccelli, N., Galzerano, G., Laporta, P., Gianfrani, L., Marangoni, M.: Comb-assisted spectroscopy of CO2 absorption profiles in the near- and mid-infrared regions. Appl. Phys. B Lasers Opt. 109(3), 385-390 (2012)

6. Fukushima, S., Silva, C.F.C., Muramoto, Y., Seeds, A.J.: Optoelectronic millimeter-wave synthesis using an optical frequency comb generator, optically injection locked lasers, and a unitraveling-carrier photodiode. J. Light. Technol. 21(12), 3043-3051 (2003)

7. Ryu, H.Y., Lee, S.H., Lee, W.K., Moon, H.S., Suh, H.S.: Absolute frequency measurement of an acetylene stabilized laser using a selected single mode from a femtosecond fiber laser comb. Opt. Express. 16(5), 2867 (2008)

8. Wu, D.S., Slavik, R., Marra, G., Richardson, D.J.: Direct selection and amplification of individual narrowly spaced optical comb modes via injection locking: design and characterization. J. Light. Technol. 31(14), 2287-2295 (2013)

9. Rohde, F., Benkler, E., Telle, H.R.: High contrast, low noise selection and amplification of an individual optical frequency comb line. Opt. Lett. 38(2), 103 (2013)

10. Sala, T., Gatti, D., Gambetta, A., Coluccelli, N., Galzerano, G., Laporta, P., Marangoni, M.: Wide-bandwidth phase lock between a CW laser and a frequency comb based on a feed-forward configuration. Opt. Lett. 37(13), 2592-2594 (2012)

11. Koke, S., Anderson, A., Frei, H., Assion, A., Steinmeyer, G.: Noise performance of a feed-forward scheme for carrier-envelope phase stabilization. Appl. Phys. B Lasers Opt. 104(4), 799-804 (2011)

12. Zhang, Z., Zhang, Z., Han, H., Wang, H., Wang, H., Shao, X., Shao, X., Fang, S., Wei, Z., Wei, Z., Wei, Z., Wei, Z.: Ultra-low-noise carrier-envelope phase stabilization of a Kerr-lens mode-locked Yb:CYA laser frequency comb with a feed-forward method. Opt. Lett. 44(22), 5489-5492 (2019)

13. Lemons, R., Lemons, R., Liu, W., de Fuentes, I.F., Droste, S., Steinmeyer, G., Durfee, C.G., Carbajo, S., Carbajo, S.: Carrier-envelope phase stabilization of an Er:Yb:glass laser via a feed-forward technique. Opt. Lett. 44(22), 5610-5613 (2019)

14. Gatti, D., Sala, T., Gambetta, A., Coluccelli, N., Conti, G.N., Galzerano, G., Laporta, P., Marangoni, M.: Analysis of the feed-forward method for the referencing of a CW laser to a frequency comb. Opt. Express. 20(22), 24880-24885 (2012)

15. Shao, X.D., Han, H.N., Su, Y.B., Wang, H.B., Zhang, Z.Y., Fang, S.B., Chang, G.Q., Wei, Z.Y.: Precision locking CW laser to ultrastable optical frequency comb by feed-forward method. AIP Adv. 9(11), 115003 (2019)

16. Telle, H.R., Steinmeyer, G., Dunlop, A.E., Stenger, J., Sutter, D.H., Keller, U.: Carrier-envelope offset phase control: a novel concept for absolute optical frequency measurement and ultrashort pulse generation. Appl. Phys. B Lasers Opt. 69(4), 327-332 (1999)

17. Di Domenico, G., Schilt, S., Thomann, P.: Simple approach to the relation between laser frequency noise and laser line shape. Appl. Opt. 49(25), 4801-4807 (2010)

18. Burkart, J., Sala, T., Kassi, S., Romanini, D., Marangoni, M.: Optical phase cloning by an integrated dual-parallel Mach-Zehnder modulator. Opt. Lett. 40(5), 816-819 (2015)

\section{Publisher's Note}

Springer Nature remains neutral with regard to jurisdictional claims in published maps and institutional affiliations. 